Aus den chirurgischen Kliniken (Prof. Dr. Berg und Prof. Dr. Á kerman) und dem Röntgeninstitut (Prof. Dr. Forssell) des k. Serafimerlazaretts in Stockholm.

\title{
Über Ulcus chronicum ventriculi et duodeni unter chirurgischem Gesichtspunkt.
}

Fine Zusammenstellung des chirurgischen Ulcusmaterials des

k. Serafimerlazaretts aus den Jahren I907-I9I4.

Von Dozent Dr. Abraham Troell.

(Mit 2 Abbildungen.)

Ein Rückblick auf die Entwicklung der Ulcus-Chirurgie in den letzten 25 Jahren gewährt ein sprechendes Zeugnis über bedeutende. Fortschritte in operativer Hinsicht wie über eine ersprießliche Entwicklung zu größerer Sicherheit beim Stellen der Indikationen. Die praktischen Errungenschaften - eine zunehmende operative Erfahrung und eine fortschreitende Verbesserung der Operationsresultate - haben unsere Kenntnisse erweitert und das Fragenstellen vertieft. Ich habe es hierbei in erster Linie auf das Kapitel von der zweckmäßigen Ausdehnung der chirurgischen Therapie abgesehen. Es war ein natürlicher Entwicklungsgang, daß, nachdem man allmählich auf klinischem Wege gelernt hatte, daß die Chirurgie bei vielen Ulcuspatienten mehr leisten kann als die interne Therapie, zunächst die Frage ins Auge gefaßt wurde, wann ein Magengeschwür und welche Art Magengeschwüre operiert werden sollen. Wie es gleichfalls ganz klar war, daß die nächste Frage lauten mußte: Welche Operationsmethode ist im einzelnen Falle am besten geeignet, ein gutes Resultat zu gewähren?

$\mathrm{Da} B$ der letztere Gesichtspunkt in späteren Jahren überall in hohem Grade der aktuelle gewesen ist, davon erhält man eine lebhafte Bestätigung sowohl an der direkt operativen Arbeit als durch 
ein Studium in der Literatur. Es liegt auf der Hand, da B die Diskussion über die Indikationen für chirurgische Therapie überhaupt jetzt gar nicht mehr die Rolle spielt, wie die Meinungsverschiedenheiten betreffs der Indikationsstellung für die einzelnen Operationen. Was die ersteren anbelangt, so ist zuzugeben, daß noch ungefähr dieselben Gesichtspunkte gelten, die Mikulicz (IIg) auf dem 26. Deutschen Chirurgenkongre 3 vortrefflich formuliert hat, oder die in den nordischen Ländern John Berg (9) etwas später darlegte. Das Neue, was seitdem hinzugekommen ist, dürfte im wesentlichen in der Erweiterung der Indikationen liegen, welche die Röntgenuntersuchung herbeigeführt hat (,Nische“ usw.). Über die Wahl einer Operationsmethode ist man dagegen bei weitem noch nicht zu ebenso großer Klarheit gekommen. In erster Linie gilt dies von der Frage nach Resektion oder Gastroenterostomie als Methode der Wahl.

Um aus klinischer Erfahrung einen Anhalt für die Indikationsstellung in der hier bezweckten Hinsicht zu erhalten, sind naturgemäß große Sericn von Operationsfällen, nach im großen Ganzen gleichartigen Prinzipien behandelt und längere Zeit hindurch nach dem stattgefundenen Eingriff verfolgt, erforderlich. In erster Linie zu dem Zweck tinen derartigen Beitrag zuwege zu bringen, habe ich eine Bearbeitung der chronischen Ulcusfälle, - 234 an der Zahl - die in den Jahren I907-I9I4 in den chirurgischen Kliniken des Serafimerlazaretts behandelt worden sind, vorgenommen ${ }^{\mathbf{1}}$ ); außerdem fand ich es bei der fortschreitenden Arbeit auf Grund der Beschaffenheit des Materials zwcckmäßig, einige Fragen, besonders betreffend die Symptomatologie und die Diagnostik einer Erörterung zu unterziehen. Während der Jahre, wo ich selbst als Assistent am Serafimerlazarett angestellt war, versuchte ich, wesentlich um einer derartigen Bearbeitung willen, soweit es möglich war, auch nach den Operationen Magensaft- und Röntgenuntersurhungen bewerkstelligt zu erhalten. Eine kleinere Anzahl Patienten habe ich Gelegenheit gehabt, nach ihrer Entlassung aus dem Krankenhause während längerer oder kürzerer Zeit zu be-

I) Alles, was der Frage von der Behandlung vón Ulcus perforans mit freier Peritonitis angehört, ist ganz weggelassen. 
obachten und zu wiederholten Malen zu untersuchen. Die große Mehrzahl derselben ist es mir im Laufe des Winters I9I6 gelungen zur Nachuntersuchung zu erhalten, wobei in mehreren Fällen dank der zuvorkommenden Beihilfe Gösta Forssells und seiner Assistenten - einige weitere Röntgenuntersuchungen gemacht worden sind.

Die über die Patienten ermittelten Auskünfte habe ich nur in dem Maße ausgenutzt, als sie meines Erachtens nach für die Ulcuskenntnis überhaupt etwas von Wert darboten. Demnach ist keine allseitige systematische Beleuchtung eines jeden Punktes in der Ulcusfrage durch meine Untersuchung zuwege gebracht, sondern es ist nur ein Versuch gemacht worden, einzelne Probleme, für welche mein Material sich besonders zu eignen schien, daraus zu entnehmen. - In bezug auf den Ursprung des Materials ist zu bemerken, daß ein sehr großer Prozentsatz der Fälle direkt aus der medizinischen Abteilung, wo Voruntersuchungen, meistens auch Röntgenuntersuchungen, gemacht waren und wo nach gemeinsamer Beratung zwischen dem Internen und dem Chirurgen die Operation beschlossen war, in die chirurgische Abteilung aufgenommen wurden. Das Material in seinem ganzen Umfang bezeichnet demnach zu einem sehr wesentlichen Teil eine Auswahl von, wie man meinte, für chirurgische Therapie geeigneten Fällen, welche während längerer oder kürzerer Zeit, eventuell wiederholte Male, ohne daucrnden Effekt Gegenstand interner Behandlung gewesen waren.

\section{Frequenz, Lokalisation, symptomatologische und diagnostische Verhältnisse.}

Was das Vorkommen des Ulcus, seine Verteilung auf die verschiedenen Geschlechter usw, anbelangt, so stellt sich heraus, daß im Operations-Ulcus-Material des Serafimerlazaretts nicht weniger als 76 Proz. der Fälle Ulcera ventriculi und nur 24 Proz. Ulcera duodeni waren. Dieses Übergewicht in der Frequenz der Magengeschwüre trifft beide Geschlechter, jedoch die Frauen mehr als die Männer. Ein Unterschied in der Verteilung der Krankheit auf Mann und Frau ist indessen vorhanden, indem 
nahezu zwei Drittel aller Ventrikelfälle auf Frauen und zwei Drittel aller Duodenalfälle auı Männer entfallen.

Für diese Berechnungen sind nur die Fälle - I75 an der Zahl - verwertbar, wo eine sichere Lokalisation des Geschwürs festgestellt werden konnte. Ausgeschlossen werden demnach eine Menge Pylorusstenosen ohne bei der Operation konstatierbarem Ulcus, wie auch einige andere Fälle, wo der operative Befund in der fraglichen Hinsicht nicht richtigr klar beurteilt werden konnte.

Von den 175 Fällen mit deutlich nachweisbarer Lokalisation waren I.33 (= 76 Proz.) Ulcera ventriculi, $42(=24$ Proz.) Ulcera duodeni. Von den ersteren kamen $48(=36$ Proz.) auf Männer, $85(=64$ Proz.) auf Frauen; von den letzteren $28(=67$ Proz.) auf Männer und I4 (=33 Proz.) auf Frauen. Von allen Männern hatten $48(=63$ Proz.) Ulcus ventriculi, 28 (=37 Proz.) Ulcus duodeni; von den Frauen $85(=86$ Proz.) Ulcus ventriculi und $\mathrm{I}_{4}(=\mathrm{I} 4$ Proz.) Ulcus duodeni. Die größere Frequenz des Magengeschwürs bei Frauen als bei Männern stimmt u. a. gut mit der Erfahrung überein, daß Sanduhrmagen meistens ${ }^{1}$ ) bei Frauen angetroffen werden; in meinem Material waren alle Sanduhrmagenpatienten (26 Stück) weiblichen Geschlechts.

Die hier gefundene gegenseitige Proportion in der Anzahl Magerund Duodenalgeschwüre - 3:I - stimmt am ersten mit den Berechnungen $K$ ümmells (IO2) (3,2:I) und der Pathologen Dietrich (8r) (3:I) und Paus ( 130 ) (3,8:I) überein. weicht aber ebenso wie viele früher publizierte Statistiken, wesentlich ab von den hohen Werten, welche englische und amerikanische Forscher für die Frequenz des Duodenalulcus gefunden haben. Vielleicht ist es von einem gewissen Interesse, einige dieser Ziffern anzugeben. Die Anzahl der Ulcera ventriculi verhielt sich zur Anzahl der Ulcera duodeni nach Trier (I77, I88), Kaspar (8I), Mathieu (I72), Burk (22), Ewald (8I), Friedrich (8I), Sommerfeld (8I), Bier (I77, I88) u. a.m. wie 9:I, 7:I, 6:I, $5: I$, I,5:I, aber nach Sherren (I66), Mayo (II2), Mayo-Robson (I77, I88), Moynihan (I77, I88) u. a. m. wie I:I,I, I:2,I:3, I:5.

Ein häufigeres Vorkommen von Duodenalgeschwüren bei Männern als bei Frauen wird von verschiedenen Seiten erwähnt [Melchior (II7), Strau $B$ und Bamberger (I72) u. a. m.].

Iür einc Abschätzung der gewöhnlichen Lokalisation von Geschwüren im Magen-Duodenum dürfte ein Material der Art wie das hier vorliegende recht wohl geeignet sein. Folgende Frequenzzahlen sind konstatiert worden.

I) Siehe z. B. Schomerus (I 70 ), Moynihan (I 70$)$, Spannaus (I 70 ), G. Petrén (r36) u. a. m. 
Ulcus ventriculi extrapyloricum $\left.{ }^{1}\right) \cdot 89$ Fälle

Stenosis pylori

Ulcus duodeni juxtapyloricum

Ulcus ventriculi (ohne nähere Präzijuxtapyloricum ${ }^{2}$ ) $4 \mathrm{I} \quad$, I $7,5^{0} \%$

$4 \mathrm{I} \quad, \quad \mathrm{I} 7,5^{0 \%},{ }_{0} \mathrm{l}$ 37 " I $6 \%$ $6 \quad, \quad 2,5 \%$

" duodeni (ohne nähere Präzi-

$$
\text { sierung im Journal) I , } \quad 0,5 \%
$$
sierung im Journal)

Fälle mit unklar. Operationsbefund 3

Summa 234 Fälle

$$
\text { I. } 5^{\circ} \text { \% }
$$

I3\%

$100 \quad 0$ \%

Ls ist bemerkenswert, daß bedeutend über dic Hälfte ron allen dem Magensacke angehörizen Geschwüren (oder Folgen von Geschwüren) außerhalb der nächsten Nachbarschaft des Pylorus gelegen haben, ebenso wie es auffallend ist, daß die ganze Anzahl Fälle mit Veränderungen im oder unmittelbar neben dem Pylorus. - im Magen und im Duodenum - nur mit kaum Io Proz. die Frequenz von Geschwüren mehr proximal im Magensack übersteigt. Diese schroffe Abweichung von der früher gebräuchlichen Auffassung von der Pylorusregion als dem Teil des Magensacks, wo das Ulcus unverhältnismäßig am häufigsten angetroffen wird, findet zum großen Teil eine plausible Erklärung in dem Umstande, daß die Diagnostik in den letzten Jahren so bedeutende Fortschritte gemacht hat. Ich habe es hierbei zunächst auf das große Plus abgeschen, das, wie sich gezeigt hat, die Röntgenuntersuchung, z. B. beim Nachweis von Ulcus curvaturae minoris, für den Kliniker bildet. (Ein sehr vollständiger Bericht über ältere und neuere Berechnungen von verschiedenen Lokalisationen des Magengeschwürs findet sich bei Harslöf (6r). Von seinem eigenen Material, 244 Ulcera an Leichen, kamen 56 Proz. auf die Curv. min., I4 Proz. auf dic Pars pylor., II Proz. auf die hintere Magenwand, 9.5 Proz. auf die vordere Magenwand, 3 Proz. auf die Cardia und 6 Proz. auf die Curv. maj.)

Die in meiner Kasuistik durchgeführte Unterscheidung der

. I) Hierin sind 7 Fälle ( $=3$ Proz. des ganzen Materials) Ulc. ventr. extrapyl. sanatum und 26 Fälle $(=I 1,3 \%$ ) Sanduhrmagen mit einberechnet.

2) Hierin sind auch die - im wesentlichen aus trüheren Jahren stammenden - Fälle eirberechnot, die im Journal als Ulcus pylori bezeichnet sind. 
Ulcera in extrapylorische und juxtapylorische schien mir sowohl unter pathologisch-anatomischem als unter klinischem Gesichtspunkt am rationellsten. Für den Kliniker ist sie es darum, weil die juxtapylorischen und die pylorischen Veränderungen sich sowohl diagnostisch - durch die Ähnlichkeit der Symptome - als auch therapeutisch - durch Übereinstimmungen in operativer Hinsicht - ganz ungezwungen von den weiter vom Pylorus entfernt sitzenden Magengeschwüren abgrenzen lassen (die extrapylorischen Duodenalgeschwüre sind, um ihrer anscheinend relativen Seltenheit willen, von untergeordnetem Interesse). Ich komme später hierauf zurück bei der Besprechung der Symptomatologie und der Diagnostik.

Als juxtapylorisches Magen- oder Duodenalgeschwür bezeichne ich - ebenso wie Kemp (87) u. a. - ein Ulcus, das innerhalb $5 \mathrm{~cm} \mathrm{Ab-}$ stand vom Pylorusring sitzt. Keiner hat mehr als William Ma yo (II2, II3, 79) die Sonderstellung betont, welche der Pylorusteil den übrigen Teilen sowohl des Magensacks als des Duodenums gegenüber einnimmt: der Endteil des Magens [Jonnescos (I42) ,canalis pyori", Forssells (50) ,canalis egestorius"] ist sozusagen ein Teil des Pylcrus, und die proximal von der Papilla Vateri gelegene Duodenumportion gehört nur morphologisch zum Dünndarm, steht aber in embryologischer, funktioneller und pathologischer Hinsicht in intimer Beziehung zum Magensack, und zwar zum Pylorusgebiet desselben.

Ncbenbei sei erwähnt, daß multiple Ulcera in etwa 5 Proz. des ganzen Materials gefunden wurden. Bei 5 Patienten ${ }^{1}$ ) wurden bei der Operation zwei offene Geschwüre vorgefunden, bei $8^{2}$ ) kam neben einem offenen Geschwüre die Narbe einer geheilten Ulcera. tion vor. Die Frequenz stimmt besser mit William Mayos (II2) und Burks (22) Bercchnungen im Jahre Igrr (etwa 6 Proz. bzw. beinahe 4 Proz.) als mit der neuerdings aus der Literatur entnommenen Angabe Schurs und Plaschkes (I62, I49), daß Magengeschwüre in $I_{3}-50$ Proz. multipel seien, überein.

Im Anschluß an diese mehr allgemeinen Gesichtspunkte in bezug auf das Vorkommen des Magengeschwïrs scheint mir das Vorhandensein anderer Bauchleiden, speziell Appendicitis und Crallenstein, gleichzeitig mit Ulcus, hervorgehoben werden

I) Fall 88 (ein Geschwür im Magen, eines im Duodenum) und (beide in Magen) 93, I41, 207, 225.

2) Fall I, 21, I29, I32, I43, I50, I59, I63. 
zu müssen. Nicht darum, weil dieses Zusammentreffen notwendigerweise eine Rolle spielen muß, sondern eher um daran $\mathrm{zu}$ erinnern, daß das Gegenteil vielleicht mindestens ebenso wahrscheinlich ist. Sowohl von chirurgischer[Hof meisters (22) Klinik, Sherren(I66)] als auch von pathologischer [Borst(I53)] Seite ist die Bedeutung der Koinzidenz dieser Krankheiten hervorgehoben worden. Rössle (I53) meint, da B Ulcus ventriculi et duodeni oftmals ein sekundäres Leiden ist. Als Stütze gibt er $u$. a. folgende Ziffern an: In den Jahren I908-I9I2 wurden unter 20 r Fällen vom offenen oder geheilten Magengeschwüren 54 gleichzeitige Appendicitiden und unter 33 gleichartigen Duodenalaffektionen 16 Appendicitiden konstatiert. Kümmell (IO2) schätzt das Vorkommen von chronischer Appendicitis bei Ulcus-duodeni-Patienten auf 75 Proz. Die Gebrüder Ma yo (Io) fanden unter ihren Ulcus ventriculi-Patienten I4 Proz., die gleichzeitig einen kranken Appendix und 5 Proz., die eine kranke Gallenblase hatten, und unter den Duodenalfällen entsprechende Zahlen mit I6 Proz. und 7 Proz. Die Frequenz von Appendicitis betrug auf das Ulcus-Material des Serafimerlazaretts nur 9 Fälle ${ }^{1}$ ), von gleichzeitigem Gallenstein nur $2^{2}$ ); unter diesen Appendicitisfällen waren nicht mehr als $3^{3}$ ) Ulcera duodeni. Die Zahlen sind so klein, daß aus denselben schwerlich eine Stütze für die Auffassung entnommen werden kann, daß die beiden in Rede stehenden Krankheiten für die Entstehung von Magen- oder Duodenalgeschwüren von ursächlicher Bedeutung sein sollten.

Unter mehr allgemeinem Gesichtspunkt bietet das Material des Serafimerlazaretts, so wie es nun vorliegt, verschiedene interessante Momente, die meines Erachtens die Symptomatologie bei Ulcus in instruktiver Weise beleuchtęn. Ein solches ist beispielsweise das Verhalten der Schmerzen. Der Wert des für die Lokaldiagnose - speziell für die Diagnose von Ulcus duodeni - großen Gewichts, das nach der Behauptung Moynihans (122, 125) u. a. m. in der Zeit des Auftretens der Schmerzen liegt, kann kaum besser erprobt werden als durch Prüfung eines einigermaßen großen
r) Fall $62,76,154, \mathbf{6} 61,175,202,212,216,224$.
2) Fall 96, I 5 .
3) Fall $154,216(?), 224$. 
Operationsmaterials, wo die Angaben der Anamnesen und die Befunde bei den Laparotomien miteinander $\mathrm{v}$ rglichen werden können. Im Anschluß daran bieten sich ungesucht verschiedene andere symptomatologische Einzelheiten zur Diskussion in bezug auf sekretorische und motorische Störungen des Magens, betreffend das Verhálten des Stuhles, betreffs der Bedeutung eines negativen, bzw. positiven Palpationsbefundes an Magen und Darm im geöffneten Bauch, eines vor der Operation palpierten Tumors usw. Die Frage, was Röntgen bei der Diagnose von gewissen Ulcusformen und -zuständen, speziell Sanduhrmagen und Ulcus duodeni, gewähren kann, gehört zweckmäßigerweise auch hierher.

In welchem Maße die Lage einer Wunde sich in dem Zeitpunkt des Auftretens der Schmerzen abspiegelt, d. h. in welchem Maße dieser letztere für die topische Diagnostik des Geschwürs von Bedeutung ist, habe ich durch die Zusammenstellungen in Tabelle $\mathrm{I}$ und $2 \mathrm{zu}$ ermitteln versucht. Alle meine Ulcusfälle haben hier nicht in Betracht gezogen werden können, teils auf Grund von unvollständigen Journalangaben, teils, weil typische Schmerzen kein markantes Symptom im Krankheitsbilde $^{1}$ ) gewesen sind.

Ich gebe $z u$, daß es nicht ganz leicht ist, überhaupt etwas einigermaßen Sicheres aus diesen Tabellen zu entnehmen, - die verhältnismäBig niedrige und untereinander sehr wechselnde Anzahl Fälle innerhalb der einzelnen Gruppen verringert die Möglichkeit gemeingültige Schlußfolgerungen zu ziehen, wie schon der Ausgangspunkt, die Angaben der Patienten von den Schmerzen, nicht allzu exakt ist. Gleichwohl scheinen gewisse Details in den Tabellen wert, beachtet zu werden. Teils das unbestreitbare Verhältnis, daß dic Patienten in diesem Material, welche über spät nach dem Essen auftretende Schmerzen, nächtliche Schmerzen und Hungerschmerzen klagten, viel öfter - in ungefähr 66 Proz. der Fälle - ein Ulcus (ventriculi s. duodeni) im oder in der Nähe

I) Andererseits kommt es in Tab. 2 vor, daß derselbe Fall mehr als einmal gezählt worden ist, wie $z$. B. Fall 233, wo bei Ulcus duodeni extrapyloricum Schmerzen nicht nur 2-3 Stunden nach dem Essen sondern auch gegen Morgen und zuweilen gleich nach dem Essen vorkamen. Tatsächlich zeigt jedoch Tab. I, daß dies auf das Endresultat nicht einwiskt, im großen ganzen werden die Prozentzahlen für entsprechende Gruppen ungefähr dieselben in beiden Tabellen. 
Ta -

Die Beziehung zwischen der Lage des Geschwürs und der Zeit für das immer oder so gut wie immer zu einem und demselben

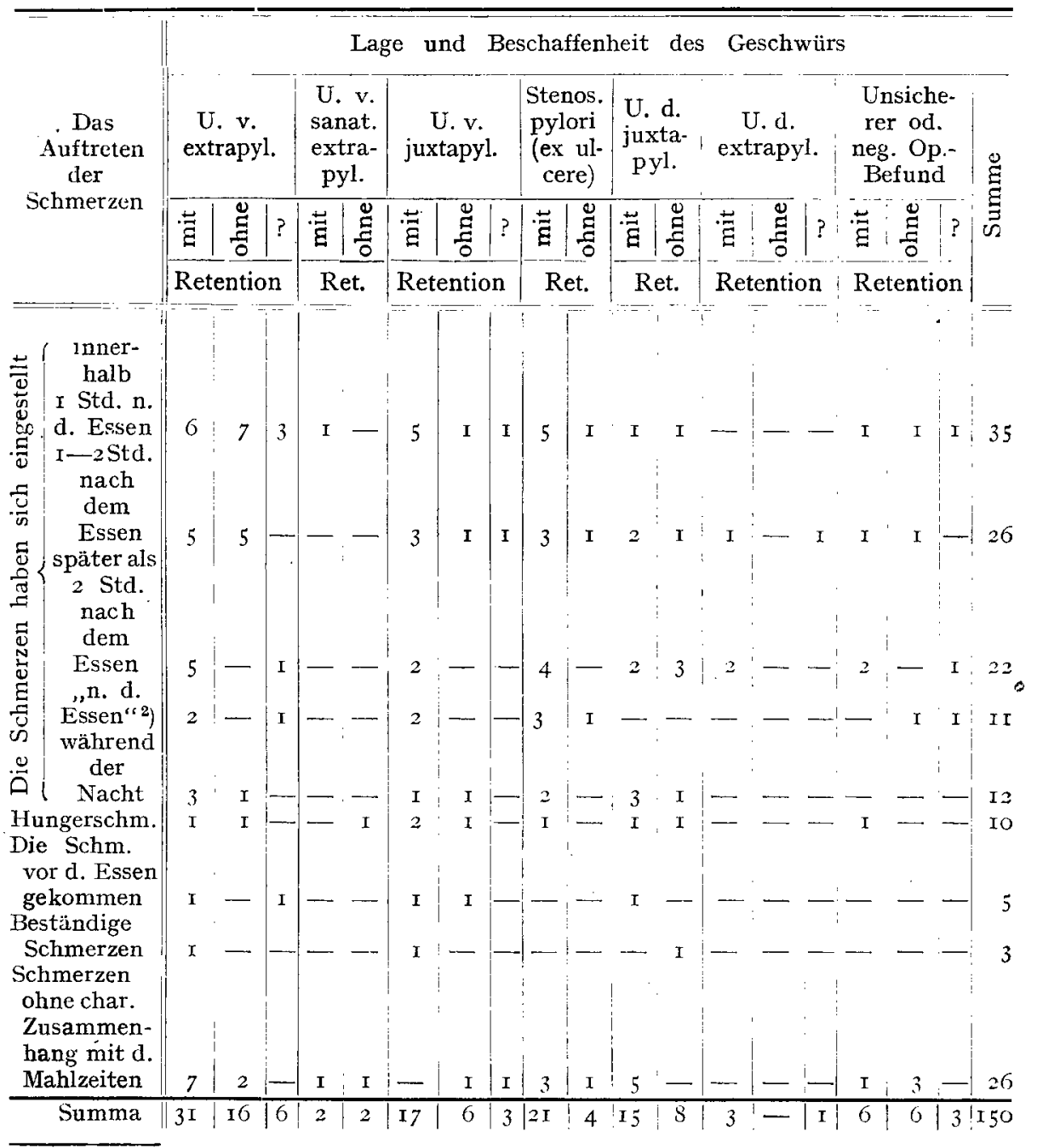

1) D. h. hier sind nur die Fälle mit aufgenommen worden, deren jeder z. B. nur Fälle sind ausgelassen worden, deren jeder Schmerzen $z$. B. sowohl 2 Stunden nach dem sondern auch die letztere Kategorie Fälle aufgenommen.)

2) Ohne exaktere Angabe oder auch bei verchiedenen Gelegenheiton vorschieden 
be 11 e 1 .

Auftreten der Schmerzen (nur die Fälle umfassend, wo die Schmerzen Zeitpunkt) im Verhältnis zu den Mahlzeiten aufgetreten sind).

\begin{tabular}{|c|c|c|c|c|c|c|}
\hline $\begin{array}{c}\text { Ulcus } \\
\text { ventriculi }\end{array}$ & $\begin{array}{l}\text { Stenosis } \\
\text { pylori }\end{array}$ & $\begin{array}{c}\text { Ulcus } \\
\text { duodeni }\end{array}$ & $\begin{array}{c}\text { Ulcus } \\
\text { ventr. } \\
\text { extrapylor. }\end{array}$ & $\begin{array}{c}\text { Ulcus } \\
\text { ventr. et } \\
\text { duodeni } \\
\text { juxtapylor. }\end{array}$ & $\begin{array}{c}\text { Ulcus } \\
\text { duodeni } \\
\text { extrapylor. }\end{array}$ & $\begin{array}{l}\text { Unklarer } \\
\text { Op.-Befd. }\end{array}$ \\
\hline
\end{tabular}

Prozentzahlen (jede der einzelnen Gruppen Fälle, eingeteilt nach der Zeit für das Auftreten der Schmerzen, kommt mit Rücksicht auf die Lage des Geschwürs in folgenden Proportionen vor).

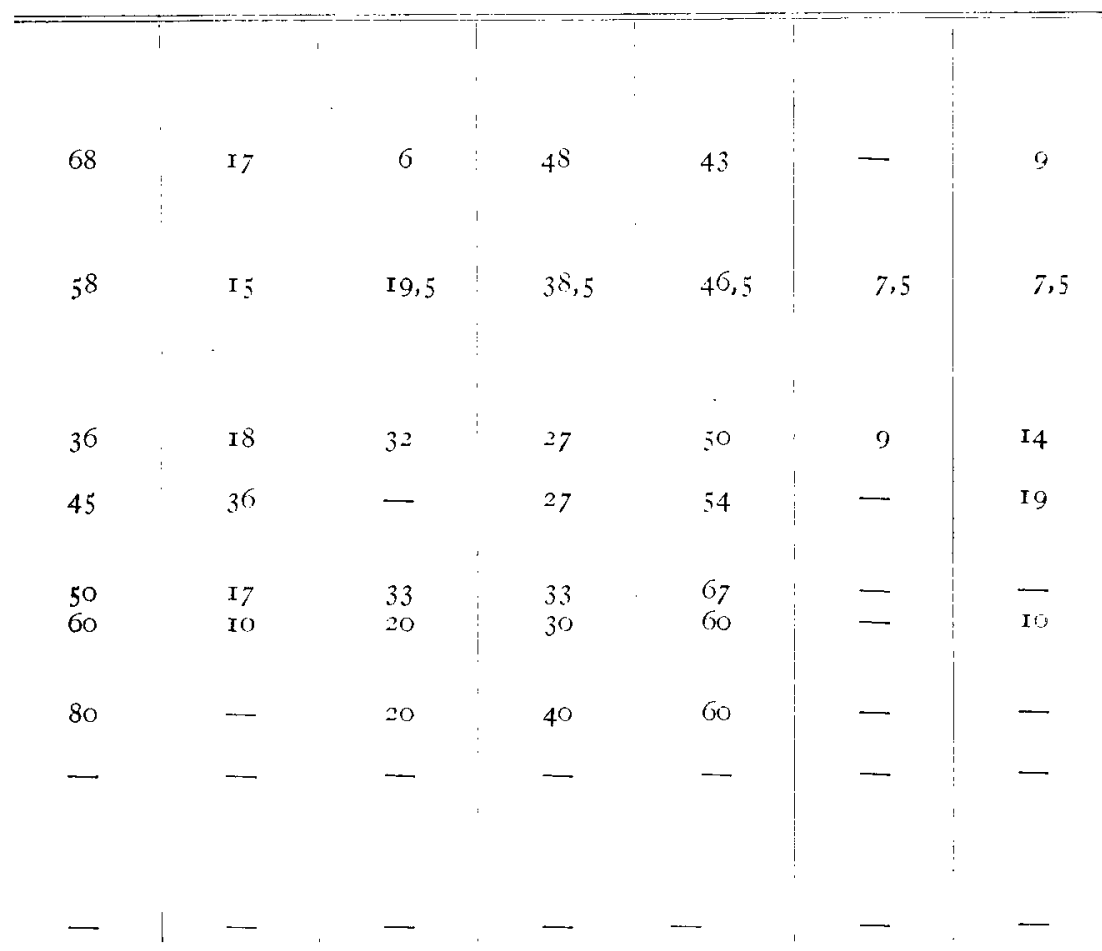

3 Stunden nach dem Essen oder nur nachts usw. Schmerzen gehabt hat, Solche Essen als auch des Nachts usw. gehabt hat. (In Tab. 2 sind nicht nur die erstere,

lange nach dem Essen. 
Ta -

Die Beziehung zwischen der Lage des (jeschwürs und der Zeit für zı gewissen Zeiten regelmäßig wiederkehrende Schmerzen vorlag; mit wenigen nommen werden, wo die Schmerzen immer oder so gut wie immer zu

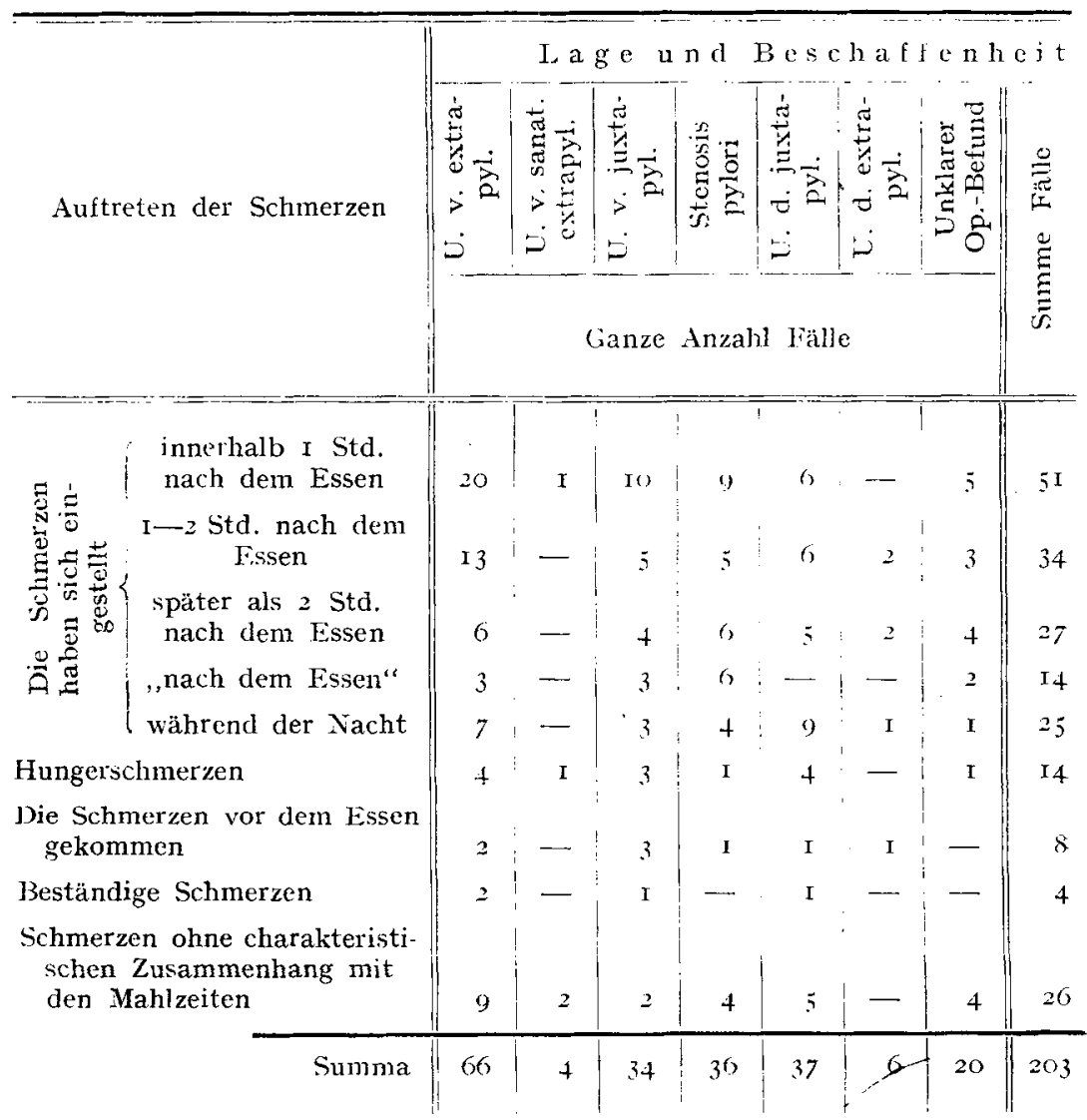

des Pylorus als ein Ulcus mehr proximal im Magensack gehabt haben. Teils aber auch der Umstand, da $\beta$ die Patienten mit bald nach dem Essen auftretenden Schmerzen in sehr großer Proportion - in gegen 5o Proz.- juxtapylorische Geschwüre auf der einen oder der anderen Seite vom Pylorusring gehabt haben. Dagegen gewährt nach den Tabellen der Zeitpunkt für das Auftreten der Schmerzen keinen für die Diagnose wertvollen, ebenso dcutlichen Unterschicd zwischen Ulcus ventriculi und Ulcus duodeni, indem sich unter den späten Schmerzfällen eine bedeutende Menge gegen 50 Proz. -- innerhalb des Magensacks belegene Ulcera und 
belle 2 .

das Auftreten der Schmerzen (alle Fälle umfassend, wo eine Angabe über Abweichungen bleiben die Prozentzahlen dieselben, wenn nur die Fälle mitgeeinem und demselben Zeitpunkt im Verhältnis zu den Mahlzeiten aufgetreten sind).

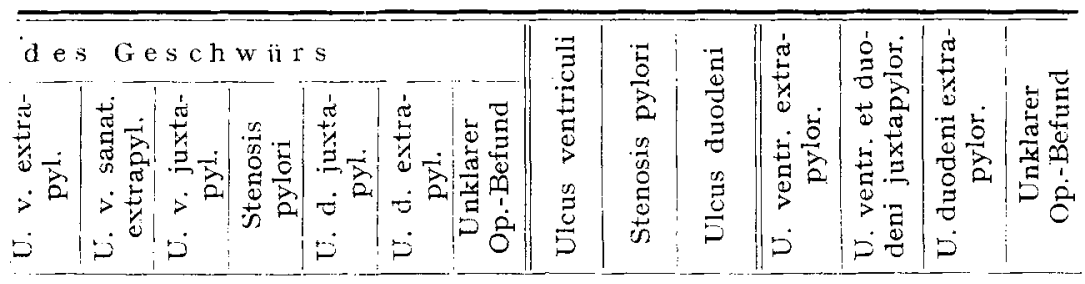

Prozentzahlen (jede der einzelnen Gruppen Fälle, eingeteilt nach der Zeit für das Auftreten der Schmerzen, kommt mit Rücksicht auf die Lage des Geschwürs in folgenden Proportionen vor).

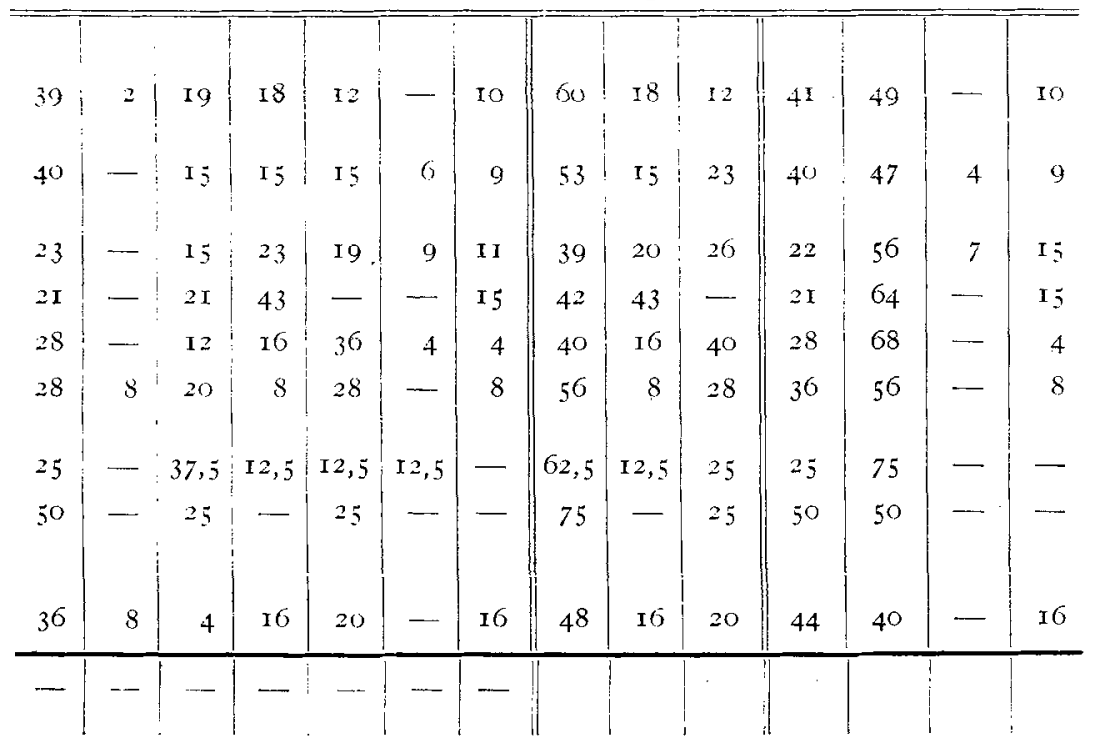

unter den früheren Sclımerzfällen nicht so wenige Ulcera duodeni kefinden. Einerseits lassen diese Verhältnisse es berechtigt erscheinen, in praktisch-diagnostischer Hinsicht die Geschwüre in der Gegend des Pylorus, sei es daß sie dem Magensack oder dem Dünndarm angehören, als eine Gruppe für sich anzusehen, selbst wenn man nicht übersehen darf, daß die Symptomatologie, wenigstens was die Schmerzen anbelangt, öfters für ein proxirnal von dieser Region gelegenes Geschwür dieselbe sein kann, wie für ein juxtapylorisches. Andererseits widersprechen die gewonnenen Erfahrungen der Richtigkeit vom Moynihans Ansicht, daß das 
Duodenalulcus unter anderen durch die Zeit des Auftretens der Schmerzen eine von dem Magenulcus leicht zu unterscheidende Krankheit sei.

Der hier berührten Frage von der lokaldiagnostischen Bedeutung der Schmerzen bei Ulcus ist in neuerer Zeit von mehreren Seiten viel Aufmerksamkeit geschenkt worden.

Die Veranlassung ist in erster Linie das Interesse, welches das Diagnostizieren von Ulcus duodeni überall geweckt hat. Die von englischen und amerikanischen Chirurgen dargelegte Meinung, daß dieses Leiden, im Gegensatz zu dem, was früher angenommen wurde, eine sehr gewöhnliche Erscheinung sei - nach Codman (30) ungefähr ebenso gewöhnlich, wie akute Appendicitis! -, muß natürlich als kräftiger Ansporn wirken, dasselbe $z u$ erkennen und zu behandeln. Und zwar um so mehr, als sie auch so äußerst typisch sein sollte, daß schon die Anamnese hinreichende und zuverlässige Anhaltspunkte gewähre[Mo ynihan (I22) ]. In dieser seien es, außer Chronjzität, Periodizität, Melaena und in der Regel Abwesenheit von Erbrechen, die Schmerzen, die eine sozusagen pathognomonische Bedeutung hätten. Sie seien mit Vorliebe etwas mehr nach rechts im Epigastrium lokalisiert und sie träten nicht früher als $2-4$ Stunden, zuweilen noch später nach eingenommener Mahlzeit auf, mit einem Maximum, wo der Kranke sich hungrig fühle (,Hungerschmerzen") oder des Nachts.

Aus diesen diagnostischen und therapeutischen Bestrebungen muBten u. a. zahlreiche Möglichkeiten hervorgehen, Moynihans Lehre zu bestätigen und eventuell zu korrigieren; in der Tat kam es zur Erkenntnis, daB die Theorie in wesentlichen Stücken irreleitend und zu schematisch sei. Noch vor ein paar Jahren wurde die diagnostische Bedeutung der späten Schmerzen von u. a. Pfannenstill (I4I), Strau $\beta$ Bamberger(I72), Sommerfeld (I72), Kümmell (IO2) u. a. m. hervorgehoben; nach u. a. Ke y (89) und K. Petrén (I37) unterläge es keinem Zweifel, daB 2-3 Stunden nach dem Essen auftretende Schmerzen meistens auf Ulcus duodeni deuteten. Indessen wurden die Einwände hiergegen immer bestimmter. Ursprünglich stammten sie, ebenso wie die Lehre selbst - für welche die Bezeichnung Bucquoy-Moynihan s (2I) Symptomenkomplex die korrekteste sei -, von französischen Autoren. Schon durch die Einführung des Ausdrucks ,syndrome pylorique" bekundeten Hartmann und Soupault (62) ihre abweichende Meinung; die Symptome rührten von einem Pylorospasmus her und seien charakteristisch für pylorische und juxtapylorische Geschwüre, einerlei, ob die Lage des Ulcus auf der Magen- oder der Duodenalseite des Pylorus sei. Im wesentlichen dieselbe Auffassung wurde von William Mayo (II2), Kemp (87), Mathieu (I08) u. a. m. ausgesprochen. In gewisser Hinsicht gleichbedeutend war $z$. B. Albrechts Erfahrung, da $B$ nächtliche Schmerzen auch bei Magengeschwüren mit Stenose angetroffen wurden, oder K üttners (I05) Meinung, daß je später die Schmer- 
zen kämen, desto näher liege das Magenyeschwür dem Pylorus, obwoh! die wirklichen Hungerschmerzen mehr für Duodenalulcus als für juxtapylorisches Ulcus überhaupt sprächen. Sommerfeld (IgI) fand spät auftretende Schmerzen in 25 Proz. bei "Ulcus ventriculi", in 69 Proz. bei „Ulcus pylori“, ihn 64 Proz. bei „Ulcus duodeni“, frïhe Schmerzen in bzw. 75, 3I, und 35 Proz. Borgbjaergs (16) Berechnungen gingen darauf hinaus, daß tardive Schmerzen zwar etwas öfter bei Duodenalals bei Pylorusulcera gefunden wurde, da 3 sie aber auch, und zwar keineswegs selten bei Ulcera in der kleinen Kurvatur außerhalb der Pylorusregion vorkämen. Für diese letztere Auffassung hat u. a. auch die spätere Erfahrung aus der Klinik der Gebrüder Mayo eine Stïtze gewährt [Graham (54)], ebenso wie eine neuerdings aus Rovsings Klinik gemachte Zusammenstellung [Wulff (I9I)].

Die SchluBfolgerungen aus der bisher vorliegenden Erfahrung müssen allem Anschein nach aus triftigen Gründen für die Hoffnung, aus dem Zcitpunkt für das Einsetzen der Schmerzen untrügliche Fingerzeige für die topische Ulcusdiagnose zu erhalten, als recht entmutigend angesehen werden. Die ron mir vorgenommenen Zusammenstellungen aus dem Serafimerlazarett dürften durch die Deutlichkeit mit der sie sprechen aus einem großen Material in einer entschiedeneren Weise als irgendwelche früheren dartun, da $B$ der Zeitpunkt der Ulcusschmerzen nicht als untrüglicher Ausgangspunkt für eine topische Diagnose genommen werden kanin, namentlich nicht betreffs des Duodenalulcus. Auf die Frage von der oftmals wertvollen Hilfe, welche die Röntgenuntersuchung für die diesbezügliche Diagnose gewähren kann, komme ich später zurück.

Angesichts der Abweichungen von der gewöhnlichen Ansicht - daß früh nach dem Essen auftretende Schmerzen wesentlich auf ein Ulcus ventriculi extrapyloricum deuten und spät einsetzende Beschwerden rorwiegend für ein juxtapylorisches Ulcus ventriculi s. duodeni sprechen - welche unter anderen Dingen aus der Erfahrung im Serafimerlazarett hervorgehen, fragt man sich unwillkürlich: Bieten die betreffenden Krankengeschichten besondere Momente dar, welche die Abweichung in dem Auftreten der Schmerzen mehr oder weniger erklären können? Die Antwort ist nicht geradezu verneinend. Prüft man nämlich die Krankengeschichten 1 ) in bezug;

I) Nur Iog Fäle sind hier verwertbar, indem für die übrigen Fälle mit.zu Deutsche Zeitschrift $\mathrm{f}$. Chirurgie. 148. Bd. 
auf die motorischen und sekretorischen Verhältnisse des Magens, so findet man, daß die Patienten mit extrapylorischen Magengeschwüren, welche später als zwei Stunden nach dem Essen Schmerzen bekamen, alle zusammen Retention gehabt haben (viermal wurde Pylorusstenosé, zweimal Słnduhrmagen gefunden), während die Mehrzahl der extrapylorischen Migengeschwüre mit frühen Schmerzen keine Retention hatten. Ferner geht hervor, dab die Totalazidität bei Ulcus ventriculi extrapyloricum mit Hungerschmerzen und bei Ulcus duodeni juxtapyloricum mit Schmerzen innerhalb I Stunde nach dem Essen durchschnittlich relativ hoch ist $(67-76$ für die erstere und $56-75$ für die letatere Gruppe Fälle - die entsprechenden Werte für die übrigen extrapylorischen Magengcschwüre und juxtapylorischen Duodenalgeschwüre sind 25-54 bzw. I3-70). Dics ber(chtigt nicht $z u$ einer sicheren Schlulßolgerung, di $B$ der Grad der Azidität oder eine Motilitätsstörung von ursächlicher Bedeutung für den Charakter der Ulcusschmerzen ist, aber es läßt als wünschenswert crscheinen, daß man bei der Untersuchung von Ulcus-P.ltienten mehr als bisher die Aufmerksamkeit auch auf diese Verhältnisse richtet. Konsequent durchg führte Untersuchungsserien müssen darüber aufklären können, inwiefern cs R $\mathrm{g}_{1} \mathrm{l}$ ist odrr nicht, $\mathrm{d} \cap \mathrm{B}$ z. B. eine ausgesprochene Hyperazidität be i extrapylorischen $\mathrm{M}-$ gonulcera mit Schmerztyp von juxtapylorischen Geschwüren und bii juxtapylorischen Duodenalgaschwüren mit Schm:rztyp von extrapylorischen Magengeschwüren vorkommt.

Wenn sich herausstellen sollte, daß die zuletzt gemachten Andeutungen in bezug auf einen besonderen diagnostischen Wert von Aziditäts- und Motilitätsuntersuchungen bei gewissen Gruppen von Magenund Duodenalgeschwüren dem wirklichen Sachverhalt entsprechen, so wäre dies von nicht geringem Werte. Die Wahrscheinlichkeit einer Bestätigung ist wohl in dieser Richtung nicht allzt gro $B$, in Anbetracht der mehr und mehr gewonnenen Erfahrung, daß die Aziditätswerte, die bei einer gewöhnlichen Probefrühstücksuntersuchung gefunden wurde, keine so entscheidende ulcusdiagnostische Bedeutung haben, wie es früher angenommen wurde - schon bei Gesunden kann der Aziditätsgrad außerordentlich stark wechseln und für die Diagnose Magengeschwür legt man nunmehr viel mehr Gewicht auf die Frage von Hypersekretion

einem gewissen Zeitpunkt auftretenden Schmerzen eine Angabe über Totalazidität oder Retention oder beides fehlte. 
als von Hyperazidität $\left.{ }^{1}\right)$. Es dürfte gleichwohl nicht unangebracht sein, in diesem Zusammenhang die Aziditätswerte bei Probefrühstücksuntersuchung anzuführen, die innerhalb der verschiedenen Gruppen Ulcus in meinem Material gefunden wurden; bei der Besprechung der Nacliuntersuchungen der Patienten komme ich noch darauf zurück.

Durchschnittlich betrug die Totalazidität (stets nach Ewalds Probefrühst ück bestimmt) für

Ulc. ventr. extrapyl. mit Retent. 32 (berechn. auf 26 Pat.)

\begin{tabular}{|c|c|c|}
\hline " & , & ohne \\
\hline , & $\because$ & ", ohne Ang. üb. \\
\hline ", & " & sanat. mit \\
\hline , & " & " ohne \\
\hline , & $\because$ & „, ohne Ang. üb. \\
\hline " & " & juxtapyl. \\
\hline & " & ohne \\
\hline & " & \\
\hline
\end{tabular}

Stenosis pyl. (ohne offenes Ulc.) mit klinisch nachgewiesener Retent. Stenosis pyl. (ohne offenes Ulc.) ohne klinisch nachgewiesene Retent.

Uilc. duod. juxtapyl.

mit ",
ohne "
nit ",
ohne ",
mit ohne ",

$$
\begin{aligned}
& 49 \text { ! " } \\
& 15(\quad, \\
& 41 \text { ( } \\
& \text { 20 ( , } \\
& 44 \text { (" } \\
& 58 \text { ( , }
\end{aligned}
$$$$
\text { " } 12, \quad)\}=35 \text { (auf } 42 \text { Fälle }
$$$$
\text { , } 1,4
$$$$
" 4 ",)=31 \text { (auf } 6 \text { Fälits }
$$$$
\text { " }
$$$$
\left.\begin{array}{rr}
, 22, & \\
, & 7,
\end{array}\right)=49 \text { (auf } 32 \text { Fälle! }
$$

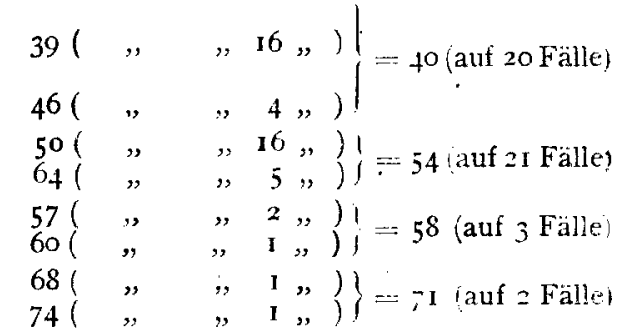

liei sämtl. Magengeschw. mit Retent. war ihr Durchschnittswert

Bei sämtl. Magengeschw. ohne Retent. war ihr Durchschnittswert

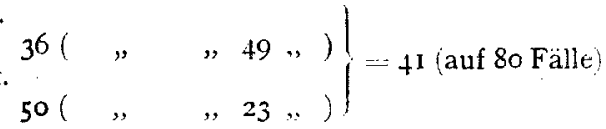

Bei sämtl. Duodenalgeschw. mit Retent. war ihr Durchschnittswert

Bei sämtl. Duodenalgeschw. ohne Retent. war ihr Durchschnittswert

Bei sämtl. juxtapyl. Geschw. u. Veränd. in Magen u. Duodenum mit Retent. war ihr Durchschnittswert

Bei sämtl. juxtapyl.Geschw: u. Veränd. in Magen $u$. Duodenum ohne Retent. war ihr Durchschnittswert

Für die Fälle mit unkl. Op.-Befund u. mit Retent, war sie

Für die Fälle mit unkl. Op.-Befund u. ohne Retent. war sie

lïr die Fälle mit unkl. Op.-Befund u. ohne Ang. über Retent, war sie

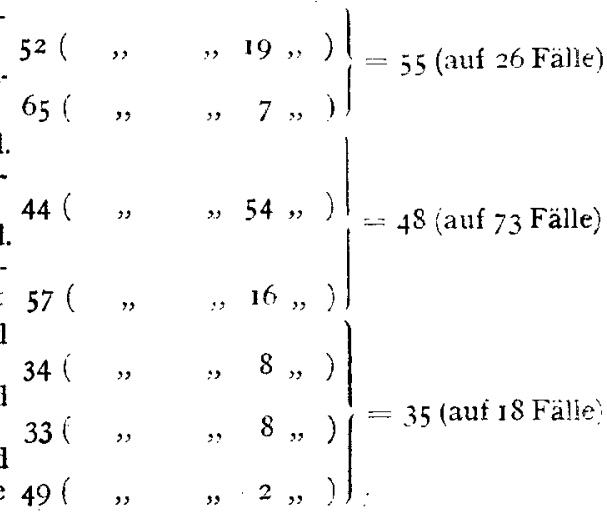

r) Ich habe keine Veranlassing hier auf diese Fragen näher cinzugehen, sondern verweise auf u. a. Michaud u. Schlecht (II8), StrauB u. Bamberger (172), K. Petrén (I37), I.indberg (I07), Borgbjaerg (I6), Amstel (2). 
Diese - durchweg auffallend niedrigen - Aziditätswerte haben wenigstens eine dokumentarische Bedeutung mit Rücksicht vor allen auf die exakte Kenntnis von der Ulcuslokalisation, welche in dem Material vorliegt. Das Vorkommen von erheblich höheren Aziditätswerten bei Duorlenal- als bei Magenulcera stimmt gut überein mit den Erfahrungen von Hyperazidität bei Ulcus duodeni, die von Einhorn (35), Viaterhouse (I25), Ma yo-Robson (II4, II6), Mayo (IO2), Kümmell (I02), $\mathrm{K}$ as par (8r) u. a.m. mitgeteilt werden. Das einzige, was daz.u hier hervorgehoben werden soll,'ist teils der Nachweis von Hypazidität oder sogar Anazidität bei manchen sowohl Duodenal- als auch vor allem Magengeschwüren, teils der durchgängige und völlig natürliche Unter schied in den Durchschnittswerten für die Fälle mit Retention und für diejenigen ohne Retention. Für die ersteren war die Totalazidität durchschnittlich 40, für die letzteren 53 (berechnet auf 84 Magengeschwüre und 34 Duodenalgeschwüre) ${ }^{1}$ ).

Es ist eine bekannte Erfahrung, daß die Aziditätsziffer, wie man sie bei Probefrühstücksuntersuchung erhält, bei demselben Individuum in mit kurzen $Z$ wischenzeiten ausgeführten Magensaftproben wechseln kann, und zwar zuweilen recht bedeutend; verschiedene Fälle in meiner $\mathrm{Zv}$ sammenstellung sind Beispiele hierfür ${ }^{2}$ ). Aber mehr der Beachtung wert sind clie Abweichungen und Schwankungen, die recht oft bei den Motilitätsuntersuchungen vorgefunden wurden. Sie kamen bei nicht weniger als 25 Patienten vor. Die Variationen an und für sich boten eine sehr bunte Mischung dar. Bald waren es Patienten, Io an der Zahl, ohne Pflaumen- oder Preißelbeeren-Retention nach 12 Stunden, aber mit einem röntgenologisch nachweisbaren Rest im Magen 4 Stunden nach dem Genuß einer Wismutmahlzeit $\left.{ }^{3}\right)$. Bald waren es Fälle mit verschiedenem Resultat bei verschiedenen Probefrühstücksuntersuchungen - zuweilen war Retention vorhanden, zuweilen nicht -aber positivem Ausschlag (d. h. Retention) bei Röntgen (4 Fälle) ${ }^{4}$ ). Diese I4 Fälle bestätigen die Erfahrung von der Röntgenuntersuchung als einer ergiebigeren Methode für den Nachweis gelinder Magenmotilitätsstörungen als die Preißelbeerenprobe bei gewöhnlicher Probefrühstückuntersuchung. Weniger gut zu verstehen sind die Fälle, wo Probefrühstücksuntersuchung, zuweilen zweimal bei demselben Patienten aus. geführt, Retention zeigt, aber Röntgenuntersuchung dies nicht tut. Dies

I) Bei so gut wie sämtlichen Magensaftproben in diesem Material ist nebst Totalaziditätsbestimmung auch die Menge freier Salzsäure festgestellt worden. Des beschränkten Raumes wegen und aus anderen Gründen wurde es nicht nötig erachtet, die diesbezüglichen Ziffern hier mit aufzunehmen.

2) $\mathrm{Nr} .3,17,40,56,103,122,182,187$ u. a.m.

3) Fall I31, 132, 159, 179, $180,186,196,208,209$. Fall 219 zeigte einmal Retention bei Röntgen, ein anderes Mal nicht.

4) Fall $135,158,170,172$. 
kam bei drei Patienten mit großem Ulcuskrater hoch oben in der hinteren Magenwand ${ }^{4}$ ), bei einem Patienten mit hochgradiger Pylorusstenose ${ }^{2}$ ) und bei einem Patienten mit Duodenalulcus dicht neben dem verengerten Pylorus $^{3}$ ) vor. Die Deutung dieser Schwankungen dürfte am einfachsten, was die drei ersten anbelangt, durch die Annahme eines Pylorospasmus gegeben sein. Analoge Verhältnisse scheinen bei solchen klinischen Typen wie Patient 86 (intermittierende Pylorusstenose mit zeitweilig bedeuten dem Erbrechen, zeitweilig keinen Retentionssymptomen) oder Fall 42 (Sanduhrmagen) oder Fall 20 r vorzuliegen. Diesen Fällen nahestehend ist wohl auch Fall 226, dessen Anamnese von Retentionserbrechen berichtet, wo aber werler Probefrühstürk- noch Röntgenuntersuchung Retention zeigte, ebenso wie die Fälle 169 und 207 (Retentionserbrechen, aber keine Retention nach Röntgen). In einer gewissen Ausdehnung ist die Auslegung denkbar, daße ein Magen, bei welchem ein Stenosehindernis angefangen hat. hohe Anforderungen an seine motorische Fähigkeit zu stellen, zuweilen, trotz der hervorgerufenen kompensatorischen Muskelhypertrophie, nicht imstande ist, das Hindernis zu überwinden; in einem noch weiter gegangenen Stadium kann er es niemals hinreichend. Eine. befriedigende Erörterung von Retentionsschwankungen bei verschiedenen Untersuchungen an demselben Individuum dürfte zur Zeit nicht möglich sein. Einzelne Fälle mit derartigen Erscheinungen sind zuvor bekannt [G.Petrén (I36), Blad (I4), Ke y (89), K.Petrén (138) u. a. m.]. Paralleluntersuchungen ähnlich denjenigen $\mathrm{Sch}$ miedens (48), Fi schers (48. und anderer betreffend röntgenologische Motilitätsstörung und klinische motorische Insuffizienz verschiedenen Grades sind sicherlich șinr wünschenswert.

lch komme auf einige weitere Gesichtspunkte in bezug auf das Verhalten der Ulcusschmerzen zurück.

Es wurde bereits angedeutet, daß bei manchen Patienten Schmerzen in der Anamnese ganz oder fast ganz fehlten. Bilden diese Fälle eine mit Rücksicht auf die Lage des Geschwürs für sich abgegrenzte Gruppe? Mit anderen Worten: gibt es Stellen im Magen und Duodenum, die dadurch gekennzeichnet werden, da $B$ hier belegene Magengeschwüre keine Schmerzen verursachen? Für die Beantwortung der Frage dürfte ein Material von chronischen Ulcusfällen weit schlechter geeignet sein als solche Fälle ron Ulcus perforans, wo die Perforation einsetzt, ohne dab früher irgendwelche Bauchschmerzen vorgekommen sind.

x) Fall $\mathrm{r} 57,220,222$.

2) Fall 138 .

3) Fall i 8 . 
Obwohl es etwas abseits von meiner tigentlichen Ulcusbearbeitumg liegt, habe ich daher die Krankengeschichten $t \in$ ils für die $32 \mathrm{Pat}-$ tienten mit Ulcus perforans, die in den Jahren Igo7-IgI4 $\mathrm{m}$ Serafimerlazarett behandelt wurden, teils für ähnliches Material ron zwei schwedischen Verfassern (G. Petrén und Norrlin) in den letzten Jahren publiziert, daraufhin durchgesehen. Nur einer der Ulcuspatienten des Serafimerlazaretts war vor der Perforation vollständig symptomfrei gewesen; das Ulcus saß bei diesem an dir Vorderseite des Magensacks dicht unterhalb der Curvatura minor (zwei andere Patienten waren während der letzten paar Wochen vor der akuten Erkrankung 1/z Stunde nach dem Essen, bzw. unabhängig von den Mahlzeiten von Schmerzen belästigt worden, das Geschwür sa $\beta$ bei diesen an der Vorderwand des Duodenums neben dem Pylorus). Unter den 99 Fällen G. Petríns (I36) von Ulcus perforans kamen 7 Fälle ohne frühere Magenbeschwerden vor: in 5 derselben handelte es sich um Duodenalgeschwüre hart am Pylorus ${ }^{\mathrm{I}}$ ), in 2 um nahe der (ardia bzw. inmitter des Fundus ${ }^{2}$ ) sitzende Magengeschwüre. Iiese säntlichen 7 Geschwürc salien an der Vorderseite des betreffenden Organs. In Norrlins (I28) Krankengeschichten über ro5 perforierende Ulcerä habe ich nicht wenicu. als Ir Fälle gefunden, wo in der Anamne se Angabe von Schmerzen fehlt - die Journalnotizen machen den Eindruck, durchaus volständig und genau zu sein. Auch in dicsen I I Fällen handelte es sich ausnahmslos um Geschwïre an der Vorderseite ron Nagen und Duodenum (abgesehen von I juxtapyloriechen ${ }^{3}$ ) und 2 (xtrapylorischen ${ }^{4}$ ) Magengeschwüren gehörten sic den dem Pylorus zunächst gelegenen Teil des Duodenums ${ }^{5}$ ) an). Daßs ts demnach wenigstens cine Zeitlang -- vollkommen latent verlaufende Ulcera gibt, legen diese Daten dar; imna $r$ ist $s$ in den von mir angezogenen Fällen die Vorderwand des betreffenden Organs gewesen. welk he

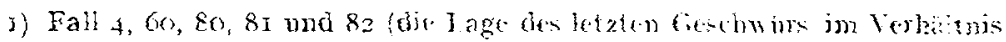
zum Pylorus nicht völlig klar).

2) Fall 44 und 56 .

3) Fall 6.

f) Fall 30,47 .

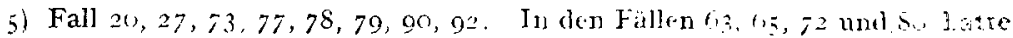
der betreffende patient $1-2$ Wochen vor der alsuten I'erforation Beschwerjen gehabt. 
der Sitz des nicht schmerzenden Geschwürs war, im übrigen scheinen sowohl die proximalen als die distalen Regionen des Magensacks in Frage kommen zu können.

Das Verhalten der Ulcusschmerzen bei verschicdenen Körperlagen wird einigermaßen durch mein Material beleuchtet, ohne daß doch die Verhältnisse so liegen, daß eine befriedigende Erklärung für die Variationen gegeben werden kann. Von größtem Interesse sind die Fälle, wo die Schmerzen durch rechte Seitenlage verschlimmert und durch linke Seitenlage verbessert wurden. Drei derselben hatten extrapylorische Magengeschwüre ${ }^{1}$, bei einem war der Operationsbefund mnklar $^{2}$ ). Drei andere Patienten empfanden unmittelbare Schmerzlinderung, wenn sie $\operatorname{sich}^{3}$ ) niederlegten oder Rückenlage ${ }^{4}$ ) einnahmen. In einem Fall (II8) von Ulcus duodeni juxtapyloricum wurden di: nachts auftretenden Schmerzen dadurch günstis becinflußt, daß der Patient aufstand.

Für 26 Fälle liegt eine ausdrückliche Angabe über Schmerzlinderung durch Einnahme von Nahrung ror. In zwei Drittel der Fälle betraf dies Magenulcera, nicht weniger als if Fälle ${ }^{5}$ ) waren Ulcera ventriculi extrapylorica. Die Erfahrungen stimmen nicht ganz mit der gewöhnlichen Auffassung, daß Schmerzen bei Duodenalgeschwüren durch Nahrung gelindert zu werden pflegen, überein, sie reimen sich besser mit W. Ma yos (II 2) Erfahrung zusammen, daß dies bei ,duodenal and antral ulcers" einzutreffen pflegt. - 38 Patienten gaben an, daß die Schmerzen durch spontanes oder hervorgerufenes Erbrechen gebessert wurden.

Von weit größerem Interesse sowohl in praktischer Hinsicht für die Diagnostik - als auch unter theoretischem Gesichtspunkt

I) Fall 58, I02 (am Pankreas fixiertes Currat.min.-Ukus), 223 (sattelförniges Curv.-min.-Ulcus).

2) Fall roz.

i) Fall 96, 159, (Ulc. ventr. extrapyl.).

4) Fall 204 (Pylorusstenose).

5) Fall 50, 54, I01, 114, 120, 182, 183, 185-I8;- I 89. Die übrigen Magengeschwüre warer: Fall $26,52,77,98,105959$. Die Duodenalgeschwüre waren Fall $13,137,140,154,172$, I 77,2 I $1,2,32$. Die Fälle fig und 10; boten unklaren operationsbefund dar. 
- un das Ulcusleiden zu verstehen - sind einige mit der Darmfunktion zusammenhängende Beschwerden, die bei manchen Patienten konstatiert wurden. Sie sind dies um so mehr, als sie einem Gebiet angehören, welchem man bisher, soweit ich habe finden können, sehr geringe Aufmerksamkeit geschenkt hat. Denn während Verstopfung von alters her als ein diagnostisch wichtiges und sehr gewöhnliches Symptom bei Ulcus ${ }^{1}$ ) bekannt ist, schien es mir unter den Fällén des Serafimerlazaretts bemerkenswert, teils daß Diarrhöe keineswegs selten vorkommt, teils daß ein Zusammenhang zwischen Stuhl und Schmerzen zuweilen deutlich bei ihnen $z u$ verspüren ist.

Bei einem Patienten mit trägemi Stuhl (Fall I9) war dieser irnmer, bei einem anderer: (Fall I78) meistens von Magenschmerzen begleitet. In einem Fall (II2) pflegte den Schmerzanfällen Verstopfung vorauszugehen, während sonst der Stuhl normal war. Bei ein paar Patienten verhielt es sich sogar so, daß die Schmerzen mit um des größerer Intensität auftraten, jc längere Zeit seit dem letzten Stuhl verflossen war (Fall I77) und gelindert wurden, sobald Stuhl sich eingestellt hatte (Fall I39). - In der Clcus-Literatur fehlt es nicht an Diskussionen über die Relation zwischen Schmerzen und Verstopfung bei Ulcus-Patienten. Unter anderem ist dic Ansicht dargelegt worden, das die Schmerzen die Ursache der Verstopfung seien [Roux $\left(I_{5} \circ\right)$ ]. Ich lasse dies im Augenblick beiseite und werde nur cin wenig bei der Frage von dem.Verhältnis zwischen den beiden Symptomen in meinen Fällen verweilen. Ich glaube, was diese anbelangt, am meisten an einen Zusammenhang analog mit dem was man besonders in Fall $\mathrm{I} 77$ - weniger bei den Patienten I. 2 und $I_{7} 8$ - aus den Operationsberichten herauslesen kann. Von

I) Ein Vermerk über dic Beschaffenheit des Stuhles ist stets im geführten Originaljournal gemacht worden, leider hat ein solches in einzelnen Fällen ganz gefehlt. Fine berechtigte Anmerkung sei hier jedoch gemacht: Die Angabe einer gewöhnlichen Krankenjournale von "Verstopfung" würde ohne Zweifel dann und wann völlig wertlos befunden werden, wenn sie von einem speziell für diese Fragen Interessierten kontrolliert werden könnte. Denn es ist eine alte Erfahrung, daß eine derartige Angabe des Patienten nicht immer ohne weiteres für unzweideutig angesehen werden kann. Um ganz exakt in der betreffenden Hinsicht zu sein, müßte das Journal genau darüber aufklären, wie oft und wann der Patient defäkiert, und über Volumen, Konsistenz des Stuhles usw. 
der Operation des ersten dieser drei Fälle entsinne ich mich gut, wie man bei Eröffnung des Bauches dadurch überrascht wurde, daß eine mächtig ausgespannte, dickwandige ColontransversumSchlinge in der Bauchwunde stark vorquoll. Diese war zu einem Teil durch feste Adhärenzen zwischen den beiden Schenkeln an der unteren Seite des Darmes in einer zusammengefalteten Lage gebunden. Die Hauptursache der außergewöhnlichen Lage und Ausspannung der Schlinge lag in kräftigen Zusammenwachsungen, die sich rom rechten Leberlappen, ron der Gallenblase und der hinteren Bauchwand nach dem Ulcusgebiet und über die obere vordere Fläche des Colon transtersum ausbreiteten. Es ist naheliegend in der starken Strammung, vor allem an den Adhärenzen zum hinteren Peritoncuin parietale, welche die Anhäufung und das erschwerte Herauspressen harter Fäkalien im Dickdarm verursachen muB, einen Anla 3 zu starken Bauchschmerzen zu sehen.

Ich gehe nun zu den Angaben über Diarrhöe in meinen Ulcusfällen über. Solcho kam bei nicht weniger als 27 Patienten während mehr oder weniger langen Perioden und abwechselnd mit Verstopfung vor.

Bei $22^{1}$ ) derselben war das U.cus nach dem Magen lokalisiert, bei 5 nach dem Duodenum (was einigermaßen dafür spricht, daß ein Magenulcus-Patient relativ öfter an Diarrhöe leidet als ein DuodenalulcusPatient - der Proportion Magenulcera zu Duodenalulcera in meinem ganzen Material 3: I gegenüber, steht das Verhältnis Magenulcus zu Duodenalulcus unter den Diarrhöefällen 5: $\mathrm{x}$ ). Nach Röntgen bot ein sehr großer Prozentsatz der Diarrhöefälle motorische Magenstörungen dar. Nur bei drei ${ }^{2}$ ) hatte sich der Magen in 4 Stundeii vollständig geleert. Bei den sämtlichen übrigen Röntgendurchleuchteten wurde ein Wismutrest übrig befunden, während außerdem stehende Curvatur-major-Einziehung $\left.{ }^{3}\right)$, gesteigerte Peristaltik ${ }^{4}$ ), organische Sanduhrverengerung ${ }^{5}$ ), (urvatura-minor-Nische ${ }^{6}$ ) und Defekt des Wismutschattens des Quer-

1) Fall $12,18,33,35,52,61,91,110,119,129,158,164,175,182,183,186$, I88, $195,205,212,213,227$.

2) Fall I+4 (völlig negativer Röntgenbefund), I95, 205.

3) Fall $6 \mathrm{r}, \mathrm{I} 86$.

4) Fall $158,167,212$.

5) Fall 129, 227 .

(1) Fall 1,: 
magens mit ${ }^{1}$ ) oder ohne ${ }^{2}$ ) sattelförmigem Ulcus in der kleinen Kurvatur konstatiert wurde.

Auf die Frage nach einem befriedigenden Erklärungsgrund für diese Diarrhöen komme ich später bei der Besprechung des Zustandes der Patienten nach der Operation zurück ${ }^{8}$ ). -

Der Frage, in welchem Grade es möglich ist, in den! geöffneten Bauch palpatorisch durch die Magenwand zu entscheiden, ob ein Geschwür vorhanden ist oder nicht, habe ich in ein paar Arbeiten über sog. Gastroptose einige Aufmerksamkeit $\left(I_{7} 8\right)$ gewidmet. Speziell habe ich dort, in Anlehnung an sehr instruktive Fälle, hervorgehoben, wie vorschnell - und zuweilen unrichtig - der Chirurg gern aus einem für die Palpation negativen Operationsbefund intra laparotomiam die SchluBfolgerung zieht, daß eine zuvor mit Röntgen für den betreffenden Fall gestellte Ulcusdiagnose falsch sein muB. Mein jetzt vorliegendes Ulcusmaterial bietet viele weitere Beispiele dar, welche die zuweilen vorkommenden Schwierigkeiten bei der Operation ein Ulcus ${ }^{4}$ ) sicher zu diagnostizieren oder auszuschließen illustrieren. Bei mehreren Gelegenheiten hat man in dem geöffneten Bauch bei Patienten, wo klinischè und röntgenologische Daten ganz bestimmt auf Ulcus deuteten, gar keine Llcusinfiltration oder -krater in der Magen-Duodenalwand palpieren können.

1) Fall 182 .

2) Fall $183,188,213$.

3) Fall $I_{1}, 63,139,144,167$.

t) Siche Fall $56,62,79$, I 39, I96 u. a. m.

(Fortsetzung dieses Artikels in Band I49, Heft I und 2 der „Deutschen Zeitschrift für Chirurgie".) 